\title{
Valoración del Capital Social en el Transporte Público y la Movilidad Sostenible en Venezuela. Una Revisión Sistemática de la Literatura
}

\section{Valuation of Social Capital in Public Transportation and Sustainable Mobility in Venezuela. A Systematic Review of the Literature}

Carmen Janeth Padrón

carmenpadron@usb.ve@0000-0003-0512-9199

Departamento de Tecnología de Servicios. Universidad Simón Bolívar- Sede Litoral.

Camurí grande-Naiguatá. Venezuela 1166.

\section{INFO ARTÍCULO}

Recibido: 20-06-2020

Revisado: 12-01-2021

Aceptado: 12-01-2021

\section{PALABRAS CLAVE}

Capital social

Transporte público

Movilidad sostenible

Venezuela

Revisión sistemática

\section{KEYWORDS}

Social capital

Public transport

Sustainable mobility

Venezuela

Systematic review

\begin{abstract}
RESUMEN
En este artículo se ha realizado una revisión sistemática entre los años 2008 y 2020 para identificar la valoración del capital social en el transporte público y la movilidad sostenible en Venezuela. Desde el punto de vista metodológico, se realizaron búsquedas en la base de datos abierta Ebscohost, encontrándose 67 estudios, de los cuales al analizarlos conforme a los criterios de inclusión y exclusión, solo 5 tenían relación con la temática. El capital social debe entederse que contribuye a la promoción del desarrollo económico, la superación de la pobreza y gobernabilidad democrática de los países. Sin embargo, en Venezuela este tiende a adquirir un carácter negativo y precario, dada la situación de crisis económica, social y política en la que se encuentra sumida este país. En cuanto al transporte público, su análisis y valoración resulta sustancial en Venezuela ya que este posee un carácter marcadamente débil, pues se constata la existencia en el país de una escasa integración de este con otros modos de transporte, a la vez que la ausencia de una movilidad sostenible.
\end{abstract}
ABSTRACT
In this article, a systematic review was carried out between 2008 and 2020 to identify the valuation of social capital in public transport and sustainable mobility in Vene- zuela. Within the methodology, the open database Ebscohost was searched, finding 67 studies and when delimiting it according to the inclusion and exclusion criteria, only 5 were related to the subject. Social capital promotes: economic development, overcoming poverty and democratic governance of the countries. Finally, in Venezue- la, social capital tends to be negative and precarious, given its economic, social and political crisis, as regards public transport its value is substantial and weak, as there is a lack of integration with other modes of transport and little mobility sustainable. 


\section{INTRODUCCIÓN}

El transporte público urbano es un servicio primordial para todos los países y sus ciudades, como enlace entre la circulación de bienes y personas, en su rol de integración social que favorece el desarrollo equilibrado de las sociedades y facilita el bienestar de sus habitantes. Este medio, no produce bienes de consumo tangibles, pero hace posible que estos se produzcan al trasladar diariamente a millones de trabajadores; no educa, pero lleva hasta los centros de estudio a miles de estudiantes; no proporciona diversión ni esparcimiento, pero apoya y hace posible el desarrollo de estas actividades lúdicas y de esparcimiento (Molinero \& Sánchez, 1998).

Esto ha generado la suficiente inquietud para realizar esta investigación, la cual tiene como objetivo general identificar la valoración del capital social en el transporte público y la movilidad sostenible en Venezuela, durante los años 2008 al 2020, mediante una concisa revisión bibliográfica sistemática. Encontrándose, dentro de la importancia del término capital social lo referente a las normas, instituciones y organizaciones que promueven la confianza, la ayuda recíproca y la cooperación en los países. Además, es parte esencial de la cultura política de una sociedad que favorece las transacciones en el ámbito económico. De igual manera, en general el transporte es un factor clave en la determinación de costos para los diferentes productos y servicios que se comercializan en un mercado cada vez más exigente y cambiante, ya que incide en el incremento de sus precios finales, generados por el traslado de los insumos, materias primas, productos terminados y personas en territorios nacionales e internacionales.

Por tanto, es una realidad que el transporte público se ha convertido en una industria estratégica, idónea para una economía mundializada y ecológica, así como para las ciudades que demandan mayores facilidades para la movilidad sustentable y segura de las personas. Este medio tiende a contaminar menos que el vehículo privado, ya que se usan menos automóviles. Consecuentemente, a través de este trabajo se ha tratado de explorar la situación actual entre los criterios de capital social, transporte público y movilidad sostenible en Venezuela, así como establecer áreas de investigación donde se necesitan revisiones sistemáticas actuales en dicho estudio.

\section{VALORACIÓN DEL CAPITAL SOCIAL EN EL TRANSPORTE PÚBLICO Y LA MOVILIDAD SOSTENIBLE}

De forma general, se puede entender el capital social según Carciente \& Varnagy (2005) como: "el conjunto de valores comúnmente aceptados que permite la sustentación y cohesión de una sociedad para que la misma pueda establecer redes de relaciones y funcionar en términos productivos, amparados" (p.13). Es importante, recordar que el "capital social" fue definido por Putnam (1993) como aquel que: "se refiere a las características de la organización social, tales como la confianza, las normas, instituciones y las redes de trabajo que pueden mejorar la eficiencia de la sociedad facilitando acciones coordinadas" (p.167). Aunque, el capital social tiene múltiples acepciones se ha de entender como aquel que se acumula al uso, facilita la vida y permite reconciliar el interés individual y, en general, como el conjunto de factores intangibles (valores, normas, actitudes, confianza y redes) que se encuentran dentro de una comunidad y que facilitan la coordinación y la cooperación para obtener beneficios mutuos.

El capital social cumple dos funciones principales. En primer lugar, ofrece beneficios económicos y sociales obvios, y se ha demostrado que fomenta el desarrollo sostenible (Collier \& Willen, 1999; Temple, 1998; Easterly, 2000), al igual que el bienestar social, la productividad y las redes sociales (Narayan \& Lant, 1999; Grootaer, 2000; Bowles, 1999; Abraham, 1985; Moser, 1996). En segundo término, produce beneficios menos tangibles, pero igualmente importantes de cohesión, unidad, sentido de pertenencia y propósitos comunes entre los ciudadanos incluso en medio de las dificultades (Seligman, 1997; Portes, 1998).

El capital social tiene tres dimensiones en cuanto que cumple funciones de aglutinante, de puente y de vinculante (Banco Mundial, 2001):

- Al juntar a personas de características semejantes para resolver un problema común dentro del grupo, el capital social aglutinante puede servir como una estrategia colectiva para el manejo de riesgos 
y formas de enfrentar dificultades. Ejemplos de este tipo, incluyen: las cooperativas de transporte público, mujeres campesinas y las cocinas comunales, entre otros.

- El capital social como puente reúne a personas heterogéneos o de diferentes orígenes en organizaciones de tipo red o en movimientos sociales de base amplia. Ejemplos de este tipo, en el transporte público incluyen: la interacción social entre los pasajeros durante el viaje en vehículos o esperar en paradas, entre otros.

- El capital social vinculante proporciona una conexión entre las personas o grupo homogéneo de familiares y/o amigos con reducido acceso a los recursos (reducción de pobreza y el desarrollo), al poder y aquellas organizaciones que, estando más allá de la comunidad, toman decisiones y controlan recursos.

Por otro lado, el capital social, se encuentra relacionado con mayor frecuencia en el análisis de redes sociales, pues pertenece a cada individuo, a veces a costa de los demás. Tal y como lo expresa Burt (1992) con simplicidad, el capital social son las relaciones sociales que uno tiene con "amigos, colegas y contactos más generales" (p.3). En su definición más simple, una red en el transporte según De Rus et al., (2007) "es un conjunto de puntos (o nodos) y líneas de interconexión que se organizan con el fin de permitir la transmisión de flujos (de mercancías, personas, información, entre otros" (p.109).

Así, cada punto puede ser un punto de origen o destino (de donde salen o a donde llegan los flujos) o un simple nodo de interconexión, cuya existencia facilita y mejora la relación entre los primeros. La existencia de redes de transporte se debe fundamentalmente a dos razones: primero, permiten una mejor coordinación de los recursos, mediante su uso sucesivo y/o simultáneo, implicando la reducción de costos, y segundo, las redes de transporte generan ventajas de valor añadido, en términos de tiempo y conveniencia de los servicios para muchos usuarios.

Hasta ahora, la investigación pudo identificar algunas valoraciones y vinculaciones débiles entre el capital social y el transporte público, así lo afirma Putnam (2000) pues hay vínculos negativos entre la dependencia del automóvil y el desarrollo efectivo del capital social e indica que dos tercios de los viajes en automóvil involucran el "conducir solo", debido a que permite la flexibilidad para escoger su propio horario de regreso, las posibilidades de llevar paquetes, la mayor protección contra el clima, privacidad o la oportunidad de escuchar la estación de radio que prefiera. Por lo que, recomienda menos tiempo de viaje y una mejor planificación de las organizaciones o comunidades para fomentar una socialización más formal. De igual forma, las dimensiones espaciales de las redes sociales han sido el foco de investigación asociada con el modelado de viajes basado en actividades. De acuerdo, con Axhausen, (2005) afirma que los teóricos de la red o los planificadores del transporte han pasado por alto o ignoran el elemento del espacio o la ubicación física de los miembros de la red, así como el aumento de los ingresos o la disminución de los costos generalizados de los viajes y cómo influyen en el comportamiento de los viajes en físico y ser socialmente excluidos Cass et al., (2005).

Otra valoración, es la Co-presencia (interacción cara a cara) que de acuerdo con Urry (2002) ha revelado el impacto significativo del capital social en la comunidad transporte y, de acuerdo con lo investigado por Cass et al., (2005) implica el contacto cara a cara (en lugar de las redes sociales online) necesario para sostener la vida social, facilitar la interacción fuerte, a través de palabras, expresiones de manos y cara, lenguaje corporal, voz, entonación y, particularmente, el contacto visual.

En cuanto, a la movilidad y el capital social Urry (2002) los valora por relación con la distancia, la frecuencia y la forma de movilidad posible ya que la reducción, canalización o limitación de ambas contribuyen a debilitar el capital social y genera nuevas formas de exclusión social. Por referencia con las ciudades habitables y el capital social según expone Vuchic (2000) aun cuando este no hace referencia directa a esta cuestión en su trabajo, en el mismo plantea la necesidad de establecer un plan de transporte para crear "ciudades habitables" ("orientados al ser humano y ambientalmente eficientes" y "económicamente viable y eficiente") y sostiene que el dominio del automóvil privado en muchas ciudades occidentales han reducido la calidad de vida en las mismas, a través de la intrusión de la contaminación del medio ambiente y la congestión en los lugares donde las personas trabajan y viven. También, este autor argumenta que la promoción 
del transporte público, caminar y el uso de la bicicleta puede mejorar la "habitabilidad" de las ciudades que él relaciona en lugares con una calidad de vida deseable, incluyendo actividades sociales, lugares públicos atractivos, cierto nivel de privacidad y un sentido de la sociedad. Un estudio que muestra un planteamiento coincidente al de este autor es el de Bunting (2004), quien vincula la movilidad con el compromiso social, activo y económico, así como la falta de movilidad con el aislamiento social.

En contraste, Shove, (2002) plantea la tesis de que a través de la movilidad no se trataría de "obtener" o ir de un punto a otro, sino que se vincularía más bien con su integración en la vida cotidiana y las actividades necesarias para su normal desarrollo (trabajo, diversión, estudios, entre otras). Por otro lado, las personas que viven en áreas rurales pueden arreglárselas sin medios de transporte y, en consecuencia, pueden llegar a casi todos los lugares que deseen. De esta manera, el capital social puede incluso verse como un bien sustituto, cuando los sistemas de transporte faltan o funcionan mal.

Otros, como Preston y Raje (2006) valoran al capital social y el transporte público con la accesibilidad y la movilidad dada la "facilidad de alcance", en oposición a la movilidad que se refiere a la "facilidad de movimiento". Ahora, se utiliza el Sistema de Información Geográfica (SIG) como herramientas útiles y versátiles de planificación al transporte público para entender, ordenar y gestionar el territorio, así como controlar parámetros en diferentes variables: mantenimiento y conservación de infraestructuras, modelar la conducta del tránsito y las dimensiones de trazado, vías aptas para el transporte, entre otros.

De igual manera, es importante estudiar ¿Cómo van a hacer los viajes con el desarrollo de las Tecnologías de la Información y Comunicación (TIC) en los Sistemas de Transporte Público Urbano? Estas capacidades tecnológicas permiten alcanzar mayor equidad en el transporte de habitantes de zonas marginadas de las ciudades, al reducir los costos y los tiempos de traslado, mejorando sus necesidades de movilidad virtual que abarca la vista de "Nuevas Movilidades".

El concepto de capital social es nuevo en lo que afecta a su vinculación con la movilidad social, que muestra las diferencias existentes en el movimiento de las personas y familias de un nivel socioeconómico u otro, con diferentes condiciones económicas y con el nivel de accesibilidad. En términos generales, Von Mentz (2003) lo definen como el conjunto de cambios económicos y sociales dados en la vida de una persona y que inciden de manera sensible en su patrimonio y en su prestigio social.

\section{METODOLOGÍA}

Esta investigación se ha desarrollado utilizando la metodología de revisión sistemática de la literatura según Petticrew \& Roberts (2006) que: "es un método explicito para resumir la información y un medio para contribuir a las respuestas y preguntas estructuradas, sobre qué funciona y qué no, y muchos otros tipos de preguntas" (p.2). La cual se realizó, mediante una búsqueda abierta de trabajos publicados en diferentes bases de datos científicas de libre acceso entre las cuales: Scielo, Dialnet, Redalyc y Google Scholar, donde no se encontraron trabajos directamente relacionados con los criterios a estudiar.

Seguidamente, se realizaron búsquedas exhaustivas en la Academic Search Ultimate de Ebscohost, considerando las publicaciones arbitradas entre los años 2008 y 2020, encontrándose 67 estudios, de los que finalmente quedaron 5 artículos que cumplían plenamente con los criterios de inclusión (Tabla 1), delimitándolos según los siguientes descriptores en idioma inglés: "Social Capital", AND "Transport" AND "Public", indicando "título a texto completo", y que fueran "publicaciones arbitradas" en "ingles", con títulos que llevaran una o varias palabras clave, antes descritas.

Posteriormente, se procedió a realizar la exclusión, que consistió en eliminar artículos duplicados, resúmenes, páginas web u otros, encontrando 38 artículos, que no estaban a "texto completo", quedando 22 no elegibles o que no cumplían con los criterios de inclusión y 6 estaban en otros idiomas, según el procedimiento para la selección de referencia o búsqueda (Tabla 2). Posteriormente se realizó, el diagrama de flujo de los estudios revisados sistemáticamente con la vinculación entre Capital Social y el Transporte Publico (Figura 1). 
Tabla 1. Publicaciones arbitradas seleccionadas.

\begin{tabular}{|c|l|c|l|}
\hline No de Búsqueda & \multicolumn{1}{|c|}{ Autores } & Año de publicación & \multicolumn{1}{c|}{ Títulos } \\
\hline 1 & Canitez & 2019 & $\begin{array}{l}\text { Los Sistemas de Transporte Público Urbano desde la } \\
\text { nueva perspectiva de la Economía Institucional: Una } \\
\text { revisión de la literatura. }\end{array}$ \\
\hline 2 & Östh et al. & 2018 & $\begin{array}{l}\text { Capital social, Resiliencia y Accesibilidad en Sistemas } \\
\text { Urbanos: Un estudio sobre Suecia. }\end{array}$ \\
\hline 3 & Rezeanu et al. & 2016 & $\begin{array}{l}\text { La influencia del urbanismo y la información. El con- } \\
\text { sumo en las dimensiones políticas de Capital social: } \\
\text { Estudio exploratorio de las localidades adyacentes a } \\
\text { la ciudad central del área metropolitana de Brașov, } \\
\text { Rumania. }\end{array}$ \\
\hline 4 & Mattisson et al. & 2015 & $\begin{array}{l}\text { Relaciones entre los desplazamientos y el capital social } \\
\text { entre hombres y mujeres en el sur de Suecia. }\end{array}$ \\
\hline 5 & Currie, \& Stanley & 2008 & $\begin{array}{l}\text { Investigando los vínculos entre el capital social y el } \\
\text { transporte público. }\end{array}$ \\
\hline
\end{tabular}

Fuente: Elaboración propia, 2021.

Tabla 2. Procedimiento de selección de referencia o búsqueda.

\begin{tabular}{|l|c|}
\hline \multicolumn{1}{|c|}{ Fase 1: Número de búsqueda en la base de datos } & Total \\
\cline { 2 - 2 } & 67 \\
\hline Total de referencias descargadas texto completo 2016-2020 & 38 \\
\hline Total Academic Search Ultimate & 22 \\
\hline Total de referencias disponibles & 5 \\
\hline Total de publicaciones académicas arbitradas & 22 \\
\hline Total de referencias no elegibles & 29 \\
\hline Duplicados & 0 \\
\hline No se ajustaba a los requisitos de idioma o fecha & 6 \\
\hline Fase 1: Total de referencias elegibles & 5 \\
\hline
\end{tabular}

Fuente: Elaboración propia, 2021.

Además, se realizó una búsqueda de la literatura gris para obtener información sobre la situación del transporte público en Venezuela, su valoración con el capital social y la movilidad sostenible, de algunos artículos arbitrados y fuentes oficiales relevantes adicionales, provenientes del Banco Mundial (BM), la Corporación Latinobarómetro, el Programa de Educación Acción en Derechos Humanos (PROVEA) o libros sobre la temática, entre otros. 


\section{Base de Datos Ebscohost}

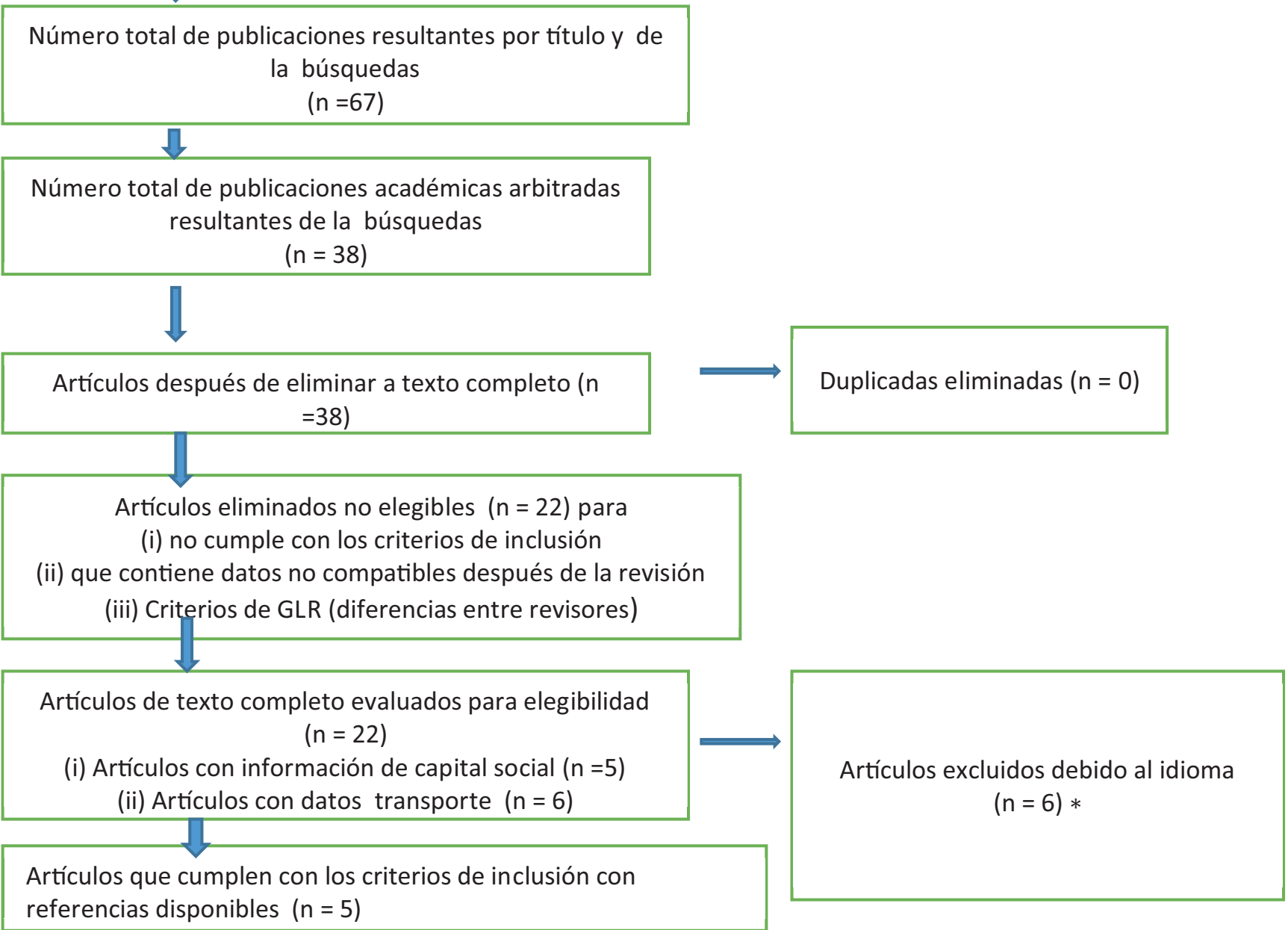

Figura 1. Diagrama de flujo de estudios revisados sistemáticamente vinculación entre Capital Social y el Transporte Público. Fuente: Elaboración propia, 2021.

\section{RESULTADOS}

Como resultados del presente estudio, se pudieron identificar pocas valoraciones directas entre el capital social, el transporte público y la movilidad sostenible de acuerdo a las revisiones sistemáticas encontradas en la base de datos EBSCOhost entre los años 2008-2020, ya que solo 5 publicaciones arbitradas cumplían con los criterios de inclusión, descritos anteriormente. Dichas, publicaciones correspondían a países de Europa del Norte (específicamente Suecia), además de Rumanía y Turquía ya que estos países generalmente cuentan con planes de transportes eficaces e integrados. Algunos realizaron análisis secundarios de datos individuales y agregados mediantes cuestionarios estandarizados, encuestas orales e incluso aplicaron el consentimiento informado previamente.

Entre las publicaciones arbitradas seleccionadas, resalta la de Currie \& Stanley (2008) ya que realizaron una investigación multidisciplinaria señalando que, aunque existen algunas conexiones indirectas con el transporte en general, el capital social es sustancial, pero existen de todos modos. También, indican que el capital social es complejo y difuso, particularmente, porque identificaron pocas investigaciones primarias cuantitativas desde una perspectiva del transporte entre el comportamiento del viaje y las redes o la interacción social. De la misma forma, indican que el transporte público es un subconjunto de opciones 
de movilidad disponibles para la comunidad y en solo pocos contextos urbanos de alta densidad, atiende una parte significativa de pasajeros, con diversas opciones de viajes, rutas, tarifas y horarios, entre otros. Seguidamente, atendiendo al objetivo general de la investigación y basado en estos hallazgos la autora realizó un cuadro comparativo de la valoración entre el transporte público y el capital social hacia la movilidad sostenible (Tabla 3).

Tabla 3. Valoración entre el transporte público y el capital social hacia la movilidad sostenible.

\begin{tabular}{|c|c|}
\hline Transporte Público & Capital Social \\
\hline $\begin{array}{l}\text { Copresencia } \\
\text { movilidad sostenible para } \\
\text { grupos desfavorecidos }\end{array}$ & $\begin{array}{l}\text { El capital social es complejo y difuso, particularmente, porque identificaron pocas } \\
\text { investigaciones primarias cuantitativas en el transporte entre el comportamiento } \\
\text { del viaje y las redes o la interacción social. El capital social aumenta la oportunidad } \\
\text { de Copresencia (Interacción o contacto cara a cara con otros) en el transporte pú- } \\
\text { blico y obteniendo como resultado una mayor movilidad. La falta de capital social } \\
\text { a menudo define los grupos sociales "en riesgo". Estos grupos en riesgo necesitan } \\
\text { de la movilidad para la inclusión social y para mejorar el capital social. El transporte } \\
\text { público proporciona viajes y está dirigido para grupos vulnerables o desfavorecidos } \\
\text { (jóvenes, ancianos, desempleados que no tienen automóvil o bajos ingresos). La } \\
\text { movilidad variara con el rango, frecuencia y formas de exclusión social. }\end{array}$ \\
\hline $\begin{array}{l}\text { Ciudades habitables y de } \\
\text { tránsito }\end{array}$ & $\begin{array}{l}\text { Las externalidades negativas del transporte público como: la congestión del tránsito } \\
\text { está haciendo que las ciudades no sean atractivas para vivir y fomentar la dispersión } \\
\text { de baja densidad. Esto puede reducir la interacción con otros. La noción plan de } \\
\text { transporte público en las ciudades fomenta la densidad de vida y hace las ciudades } \\
\text { habitables. Esto fomenta una mayor interacción social, particularmente haciendo } \\
\text { puente con el capital social. En cambio, la dependencia del automóvil crea un dete- } \\
\text { rioro en el capital social. }\end{array}$ \\
\hline $\begin{array}{l}\text { Viajar con otros e interacción } \\
\text { social } \\
\text { Auto compartido } \\
\text { Viaje en Taxi }\end{array}$ & $\begin{array}{l}\text { El capital social implica redes o puentes sociales con otras personas, por lo que viajar } \\
\text { en vehículos de transporte público o esperar en paradas / intercambios. Fomenta la } \\
\text { interacción social con personas que no se conocen. Así como, la interacción con los } \\
\text { conductores de autobuses o taxi pueden facilitar o debilitamiento del capital social. }\end{array}$ \\
\hline $\begin{array}{l}\text { Otro } \\
\text { Accesibilidad } \\
\text { Percepciones de crimen / mie- } \\
\text { do y transporte público } \\
\text { Movilidad Social y virtual (TIC) } \\
\text { Ferrocarril }\end{array}$ & $\begin{array}{l}\text { En relación, al capital social puede incentivar la buena accesibilidad (facilidad de } \\
\text { alcance), aunque no se encontraron referencias directas en la literatura entre estos } \\
\text { conceptos (en oposición a la movilidad "facilidad de movimiento"). Sin embargo, la } \\
\text { accesibilidad cuando es problemática genera exclusión social y el capital social es } \\
\text { negativo. Una barrera para el uso del transporte público (siniestros viales, evasión } \\
\text { de tarifas, crimen, miedo u otro) puede reducir el potencial de desarrollo del capital } \\
\text { social. Si la confianza interpersonal está deteriorada en las instituciones del trans- } \\
\text { porte público, debilita el capital social. } \\
\text { Utilizar las TIC en el transporte público aumentar el contacto social y la movilidad } \\
\text { virtual. } \\
\text { El concepto de capital social es nuevo en la movilidad social. Actualmente, se requie- } \\
\text { re de una movilidad virtual en el transporte público: aplicaciones de pago, billeteras } \\
\text { electrónicas, pagos con QR y más recientemente la anunciada posibilidad de usar } \\
\text { las tarjetas de crédito o débito por puntos inalámbricos u otros aumenta el capital } \\
\text { social. Los trabajadores ferroviarios tienen una cultura de ser parte de una organiza- } \\
\text { ción y pueden relacionarse con sindicatos y puede unirse con el capital social. }\end{array}$ \\
\hline
\end{tabular}

Fuente: Elaboración propia adaptado de Currie \& Stanley (2008).

Asimismo, estos autores indican que los pasajeros del transporte público son particularmente los grupos desfavorecidos (los jóvenes de bajos ingresos, ancianos, desempleados), que por definición no tienen automóviles y tienden a carecer de capital social, movilidad personal y accesibilidad. Finalmente, como se pudo observar en esta tabla el transporte público puede proporcionar movilidad para este grupo desfavorecidos, y al hacerlo, brindar una mayor oportunidad para crear redes sociales o aumentar la copresencia, la confianza interpersonal y reciprocidad. En este sentido, la falta de acceso al transporte público y la exclusión social debilita el capital social. Por otro lado, no se identificó relación explícitamente con la movilidad social y la 
accesibilidad, aunque son aspectos relacionados con las redes sociales. El capital social es un objetivo más sustantivo para lograr la equidad social, ciudades habitables y mejorar la calidad de vida de sus habitantes.

De la misma manera, otros autores como Canitez (2019) afirman que el papel del Sistema de Transporte Urbano (STU), la Nueva Economía Institucional (NEI) y las redes sociales no se han cubierto adecuadamente en la literatura, importantes para comprender el papel de las asociaciones de operadores del transporte público, especialmente en ciudades en desarrollo y aliviar los problemas de acción colectiva. Los STU son complejos incluyen lo social, económico, político, tecnológico e involucran a una variedad de actores del transporte, como las autoridades reguladoras, operadores (conductores) y pasajeros. Por lo tanto, este estudio determinó que las instituciones informales (vehículos particulares) del transporte público colectivo no están afiliados a empresas legalmente constituidas como el transporte comunitario, con elementos como: capital social, confianza, cultura, normas, rutinas, tradiciones, costumbres y hábitos. Por consiguiente, estas instituciones, toman mucho tiempo para cambiar; por lo que requieren enfoques de planificación del transporte a largo plazo.

En este contexto, Östh et al.(2018) lograron examinar la resiliencia (capacidad de un sistema, en presencia de un choque) de los sistemas de redes espaciales y sociales en relación con su funcionamiento endógeno, su provisión de capital social y su accesibilidad determinando que son factores críticos y complejo, dado que su evolución está co-determinada por la: proximidad del mercado, desarrollo del mercado laboral y de la vivienda, servicios públicos, uso y acceso a los sistemas de transporte, composición socioeconómica de la población, entre otros. En esencia, el capital social se origina en la creación y mejora de las redes sociales y la confianza entre amigos, colegas, vecinos y otros. Esta valoración, es lo que se llama capital social puente. De igual manera, llegaron a los siguientes resultados: un aumento de la accesibilidad corresponde a un aumento del capital social, la edad tiene un efecto negativo en todas las cohortes, con excepción de un impacto positivo para personas adultas jóvenes entre los 18 y 29 años.

De igual forma, Rezeanu et al. (2016) señalan que los indicadores para medir el concepto de urbanismo y su relación con las dimensiones políticas del capital social: área de superficie de la localidad, densidad de vida, necesidad percibida de resolución de problemas están asociados con la infraestructura del transporte por carretera dentro de la localidad, estatus administrativo como localidad urbana, distancia de la casa del encuestado al centro de la ciudad central, número de unidades de servicios públicos dentro de la localidad y densidad de la red de infraestructura para el acceso a servicios públicos. Coincidiendo, que el concepto de capital social es mucho más complejo que sus dimensiones políticas.

Por otro lado, Mattisson et al. (2015) examinaron la relación entre los modos de transporte, tiempo de desplazamiento, la participación social, la confianza general con otras personas no se asocian significativamente con las medidas disminuidas del capital social excepto entre los viajeros de larga duración, pues prevalece una menor participación social, y donde más mujeres que hombres se desplazan activamente en el transporte público menos de 60 minutos. El capital social es un recurso que surge de las relaciones sociales en una sociedad que puede utilizarse para resolver problemas de carácter individual o colectivo. Por otra parte, determinaron, que la participación social representa la parte estructural del capital social, encontrando que hay una baja participación social de $27 \%$ para las mujeres y $32 \%$ los hombres. A la vez, el $35 \%$ de las mujeres y el $33 \%$ de los hombres informaron una baja confianza general. Concluyendo, que la dependencia del automóvil disminuye el capital social.

La revisión literaria realizada para examinar la situación de transporte público en Venezuela y su valoración con el capital social y la movilidad sustentable, es sustancial y débil, ya que a través de algunos artículos arbitrados y la literatura gris señalada, dichos hallazgos determinaron que el Sistema de Transporte Urbano Publico (STPU) que se presenta en Venezuela desde la década de los años 80, con base en el vehículo particular, tiene serias consecuencias de externalidades negativas para las ciudades de acuerdo con el Programa Venezolano de Educación Acción en Derechos Humanos (PROVEA, 2016) como: "presenta importantes rasgos de informalidad, que se observa en la forma de la explotación del servicio, siendo más artesanales que empresariales, congestión crónica, desarticulado, atomizado, sin mantenimiento e integración y aumento de los siniestros viales" (p.8), lo cual tiene grandes repercusiones en el nivel del servicio y en los costos de operación. 
Además, el STPU en Venezuela presenta deterioro de la red vial existente, ante la incapacidad financiera del Estado para dotar de flotas nuevas de vehículos destinados para el transporte público, espacio para la circulación y el estacionamiento de vehículos, aunado a la crisis económica, social y política que predomina ante la insensibilidad frente a las exigencias de las clases sometidas, sin protección alguna, a la intemperie de la competencia, al que se demanda el mantenimiento de equilibrio económico general y la persecución a fines de justicia social (lucha contra la pobreza, redistribución de la riqueza, tutela de los grupos sociales más débiles, insostenibilidad y colapso de los servicios públicos: luz eléctrica, agua potable, transporte público, y la infraestructura del transporte, entre otros). Todo esto, genera disminución de la movilidad en la población, lo que ocasiona que haya desigualdad social en el mismo, y debilita el capital social.

En Venezuela, al igual que en otros países, la responsabilidad última de la provisión de los servicios de transporte público recae en el Estado (monopolio público) según lo señalado por Padrón (2020) "a través del Ministerio del Poder Popular para el Transporte Terrestre (MPPTT), encargado de trazar las políticas públicas del transporte terrestre y del Instituto Nacional de Transporte Terrestre (INTT), de llevar el Registro Nacional de Transporte Terrestre y gestionar estadísticas y dispositivos para el control del tránsito" (p. 3), entre otros, tanto en el ámbito local como regional o nacional, ya que se considera que los ciudadanos y las empresas tienen un derecho a la movilidad a lo largo del territorio, dicho derecho debe garantizarse por parte del sector público.

Por otro lado, en Venezuela según Padrón (2020) concluyo que el STPU tiende a ser negativo e ineficientes, presenta caída en la movilidad y accesibilidad, lo que disminuye el capital social, debido a que ocasiona desigualdad social en el transporte, siendo demostrado al "implementar unos vehículos 350 de carga pesada dada la profunda crisis del transporte público en el año 2018, denominados popularmente por el pueblo o usuarios como "Perreras" o "Guaguas" (p.7), en el territorio nacional y en el estado Vargas, por la Alcaldía del Municipio Vargas (Figura 2) así como otros tipo de vehículos (pickup y otros) no aptos para trasladar pasajeros y que no fueron autorizadas por el (INTT), ni tipificados en la Ley de Transporte Terrestre vigente del 2008, y menos en su Reglamento que data de 1998.

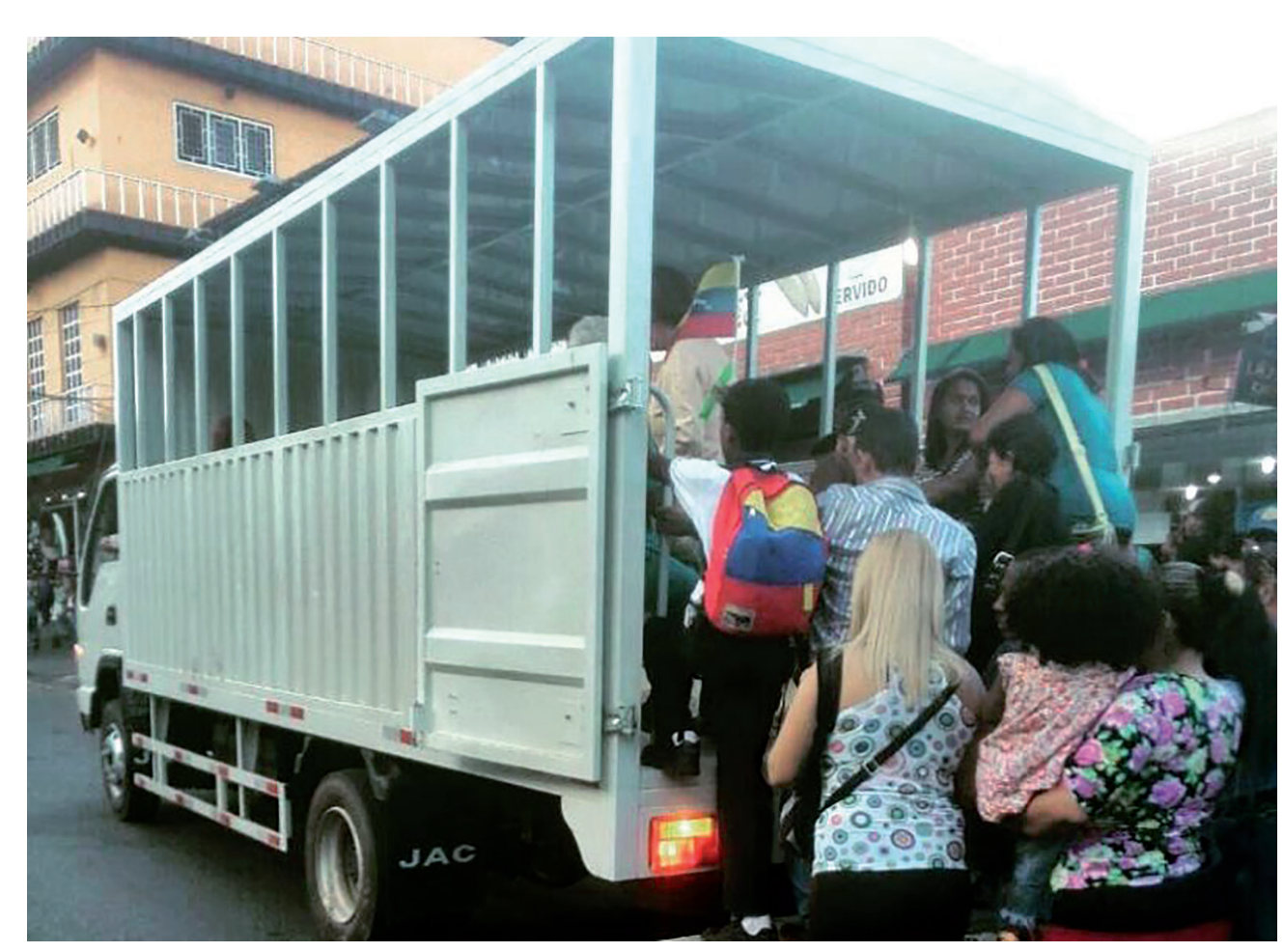

Figura 2. Vehículo de carga llamado “Perreras”. Fuente: Machado, S. (2018). 
Por otro lado, en la mayoría de las ciudades venezolanas los desplazamientos se realizan siguiendo una distribución de $60 \%$ en vehículos públicos y $40 \%$ en vehículos privados, ocupando este último la mayor parte de las vías de circulación.

En lo que respecta, a los datos relacionados directamente con el capital social en Venezuela, refleja un impacto negativo, de acuerdo con la información proveniente del Informe (LATINBAROMETRO, 2018) de América Latina, considerada esta organización como la segunda base de datos más utilizad, luego del World Values Survey, en dicho informe aplicaron 20.204 entrevistas, con muestras representativas de la población total considerando para este estudio algunos indicadores relacionados con el objeto de estudio, como: la mala situación económica actual de los países de América del Sur, confianza interpersonal, satisfacción con la vida, ingreso subjetivo, sin suficiente comida para alimentarse y clasificación de la clase social. Por lo que surge el interrogante: ¿Cuán mala tiene que ser la situación económica de un país para declararlo con crisis económica?, para obtener dichos datos consideraron: la tasa de crecimiento económico, el coeficiente Gini, la inflación para medir su desempeño, entre otros.

Por lo tanto, la economía en América Latina es brillante para algunos, no para todos. En estos cuatro países, independientemente de lo que muestran los indicadores económicos, más de la mitad de la población reconoce una mala situación económica, siendo un factor socioeconómico relevante que incide negativamente en el capital social Gráfico 1).

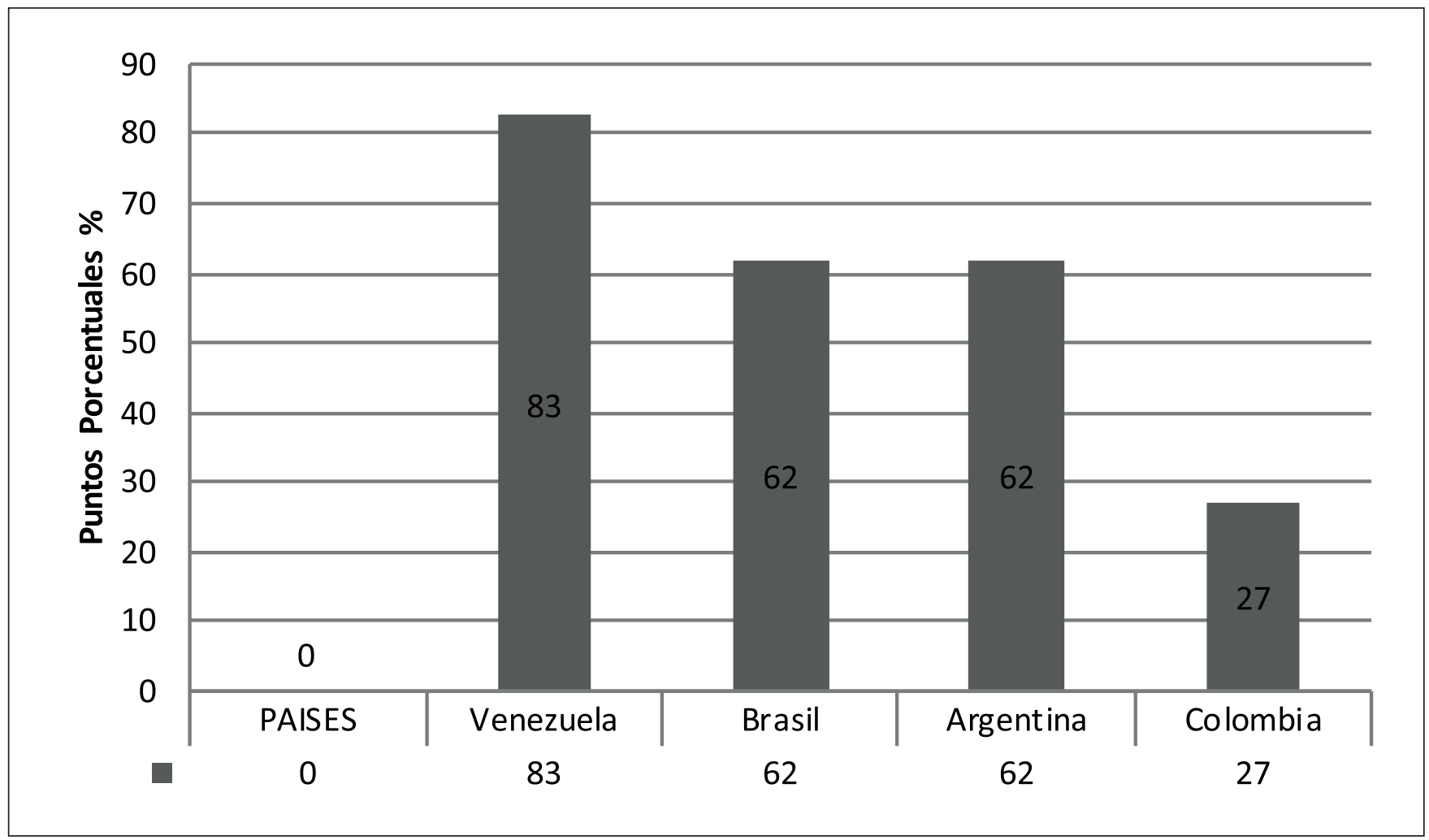

Gráfico 1. Situación Económica América Latina (países seleccionados) 2018. (En porcentajes). Fuente: Elaboración propia sobre la base de los resultados de LATINBARÓMETRO (2018).

Venezuela, en primer lugar, con el 83\%, donde los venezolanos opinan que la situación económica actual es mala, predomina una profunda crisis (recesión) dada la inflación que ha pulverizado el salario real y el poder de compra de los venezolanos, lo que debilita negativamente el capital social, seguidamente esta Brasil y Argentina con el 62\%, con crisis cambiarias y fuertes desbalances macroeconómicos, en segundo lugar. Mientras que, Colombia con el $27 \%$, ha logrado diversificar su economía a lo largo de los años. 
De igual forma, se estudió la confianza hacia todo tipo de instituciones, específicamente la confianza interpersonal. El problema con la confianza en instituciones es que en 2018 , no se registraron aumentos, lo que debilita el capital social (Gráfico 2).

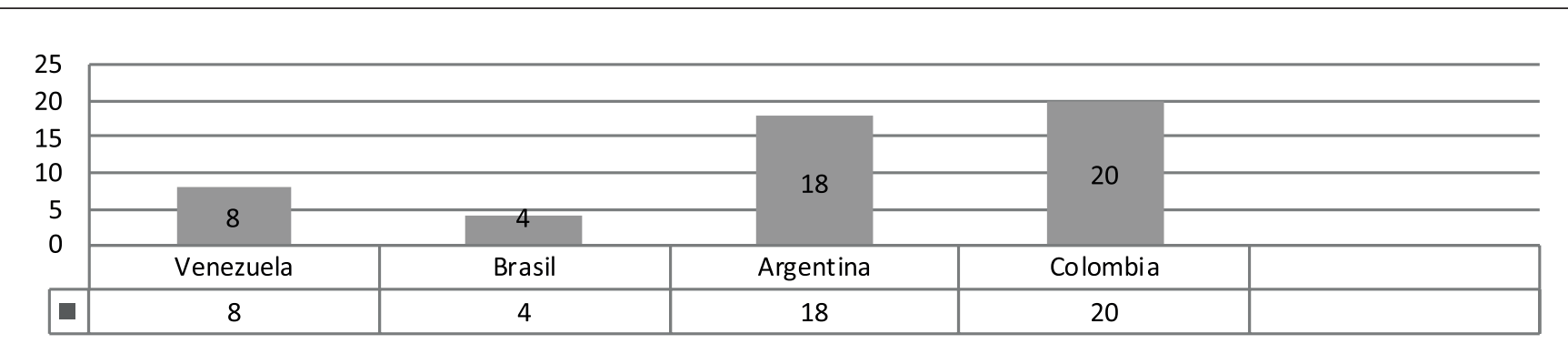

Gráfico 2. Confianza Interpersonal América Latina (países seleccionados) 2018. (En porcentajes). Fuente: Elaboración propia sobre la base de los resultados de LATINBARÓMETRO (2018)

En este informe, se observa que la confianza interpersonal no registra aumentos, siendo la región del mundo más desconfiada de la tierra, en Brasil registra 4\%, Venezuela solo alcanza el 8\%, pues predomina la desconfianza en sus instituciones, lo que debilita el capital social, luego le sigue Argentina $18 \%$ y Colombia $20 \%$, ya que sus clivajes principales, es decir tienen problemas que acusa divisiones en las sociedades y que impiden su integración y no han sido solucionados hasta ahora.

En cuanto, a la satisfacción de vida en América Latina es una de las regiones más felices de la tierra, sus grados de satisfacción de vida en promedio son altos, lo que aumenta el capital social (Gráfico 3).

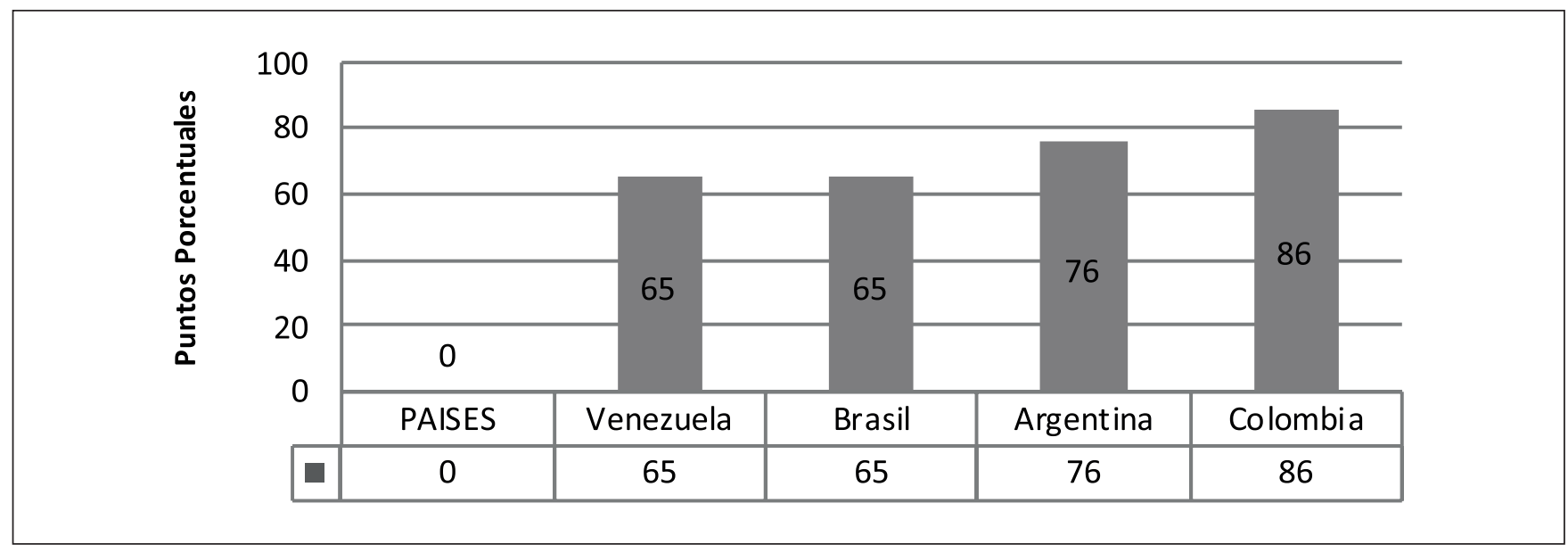

Gráfico 3. Satisfacción de vida en América Latina 2018. (En porcentajes). Fuente: Elaboración propia sobre la base de los resultados de LATINBARÓMETRO (2018).

La satisfacción de vida en Venezuela ha perdido doce puntos porcentuales entre el año 2015 y 2018 bajando de $77 \%$ a $65 \%$, siendo no muy satisfecha, lo que debilita el capital social. En todos los países de la región más de la mitad de la población está satisfecha con su vida. El país más satisfecho en este caso es Colombia con $86 \%$.

Otro indicador, que se tomó en cuenta fue el ingreso subjetivo o sueldo en América Latina, el cual se define como un indicador que muestra la evolución de las necesidades satisfechas de ingreso de la población 
y la manera como este alcanza o no para llegar a fin de mes (Gráfico 4). En todos los países de la región más de la mitad de la población está satisfecha con su vida.

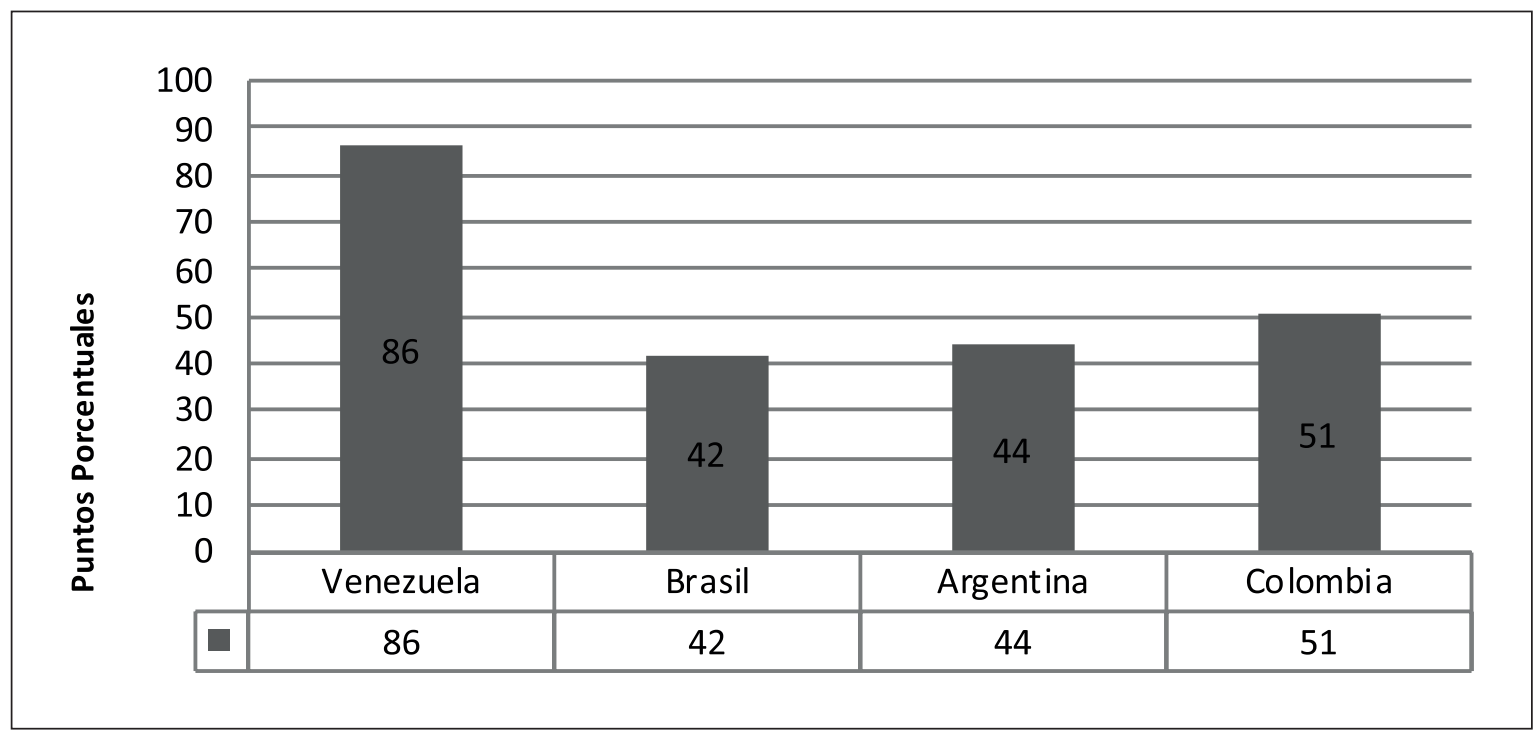

Gráfico 4. Ingreso Subjetivo o sueldo en América Latina 2018. (En porcentajes). Fuente: Elaboración propia sobre la base de los resultados de LATINBARÓMETRO (2018).

El ingreso subjetivo o sueldo en Venezuela no les alcanza, tienen grandes dificultades, el cual se ubicó en el $86 \%$ en el 2018, seguidamente se encuentran entre los cinco países (Colombia (51\%), Argentina (44\%), y Brasil (42\%) que tienen más de la mitad de su población que tiene dificultades de ingreso, lo cual es considerada como una característica negativa y precaria para el capital social.

Por tercer año consecutivo, Venezuela registra 8 de cada 10 venezolanos no les alcanza el sueldo, generando, la crisis económica y alimentaria que abarca a casi toda la población. En efecto, si se analiza los más vulnerables o sin suficiente comida al día para alimentarse, en los últimos años la región no ha podido avanzar en el desmantelamiento de la pobreza (Gráfico 5).

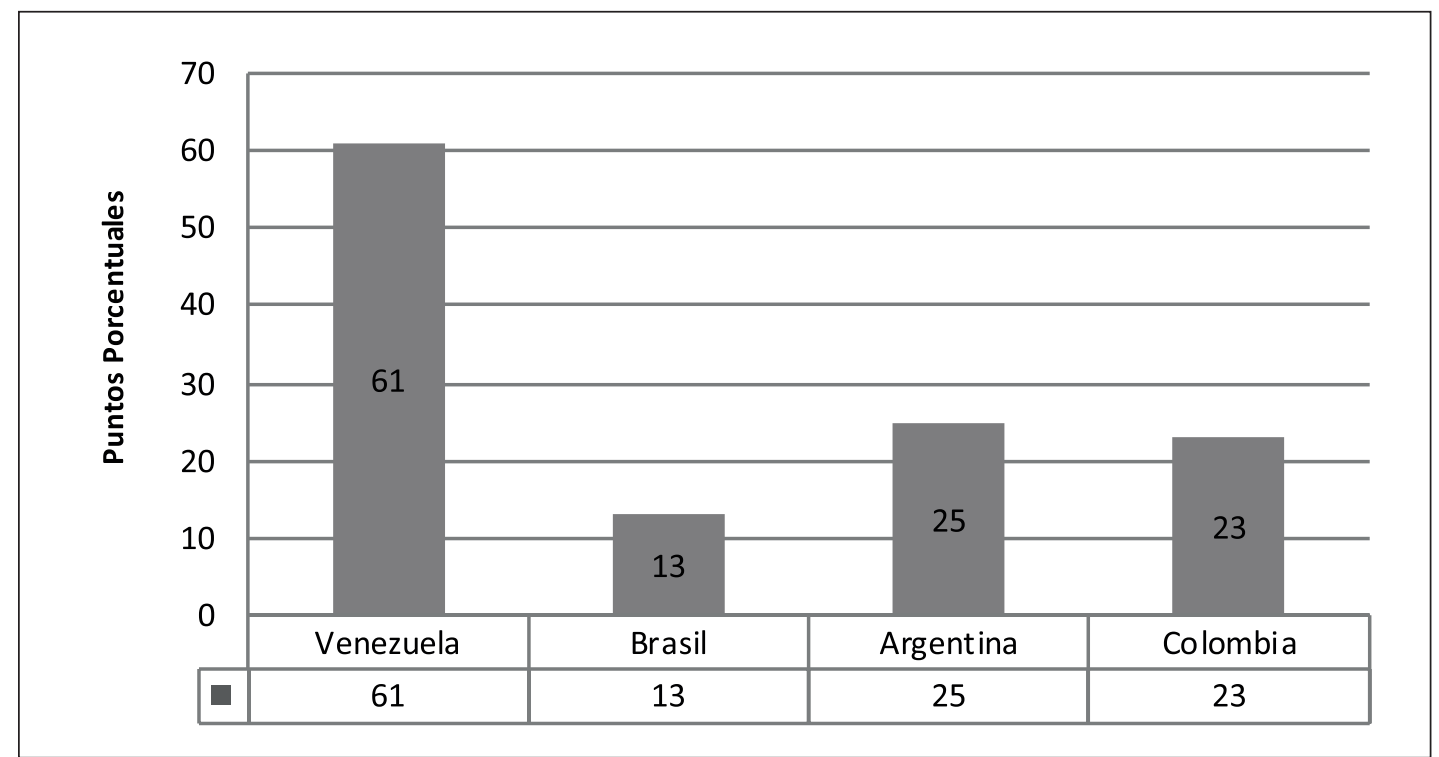

Gráfico 5. Sin Suficiente Comida para Alimentarse en América Latina 2018. (En porcentajes). Fuente: Elaboración propia sobre la base de los resultados de LATINBARÓMETRO (2018). 
Desde 2015, viene aumentando la cantidad de personas que declaran no tener suficiente comida de $22 \%$ a $27 \%$ en 2018, en el último año solamente aumenta tres puntos porcentuales de $24 \%$ en 2017 a $27 \% 2018$. Un $61 \%$ de los venezolanos declaran no tener suficiente comida al día, por lo que su capital social es negativo y precario. Dentro de los otros países, esta Argentina con un 25\%, Colombia 23\%, y Brasil 13\%. Aquí es donde está el verdadero problema de fondo de los países de Centroamérica. Se trata de una población donde un tercio o más no tienen suficiente comida para alimentarse. Todos los otros problemas son derivados en gran parte de este problema central.

En lo que respecta, a la clase social (Gráfico 6). En los años 2017 y 2018 en Venezuela, aumenta la clase baja a $62 \%$, al igual que su ingreso, especialmente en las personas más vulnerables de la sociedad, por lo que su capital social tiende a ser negativo y precario.

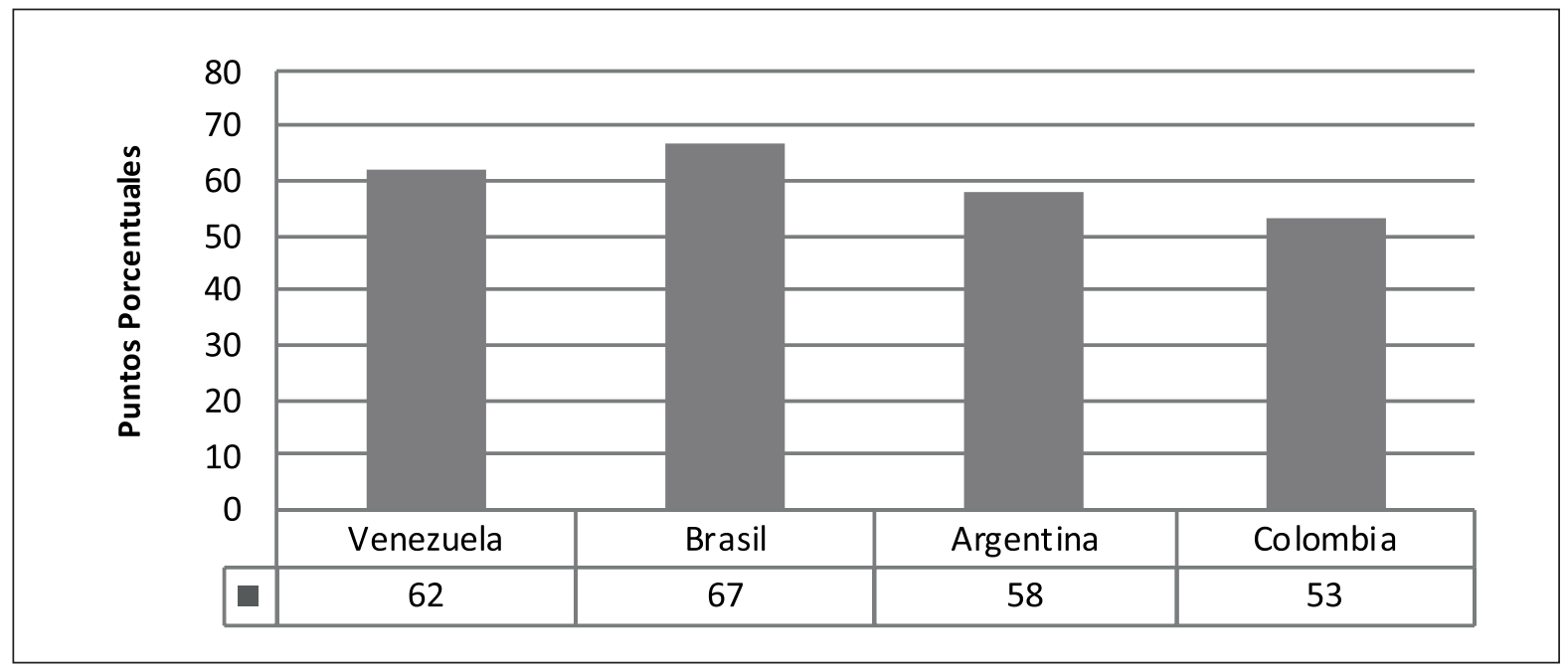

Gráfico 6. La Clase Social en América Latina 2018. (En porcentajes). Fuente: Elaboración propia sobre la base de los resultados de (LATINBAROMETRO, 2018).

En este sentido, se observa que en los cuatro países de la región hay una disminución significativa de la clase alta y media entre 2013 y 2018. La mayor caída se produce en Brasil $67 \%$, en segundo lugar, Venezuela, donde cae $62 \%$, seguidos de Argentina $58 \%$ y Colombia $53 \%$ en ese período.

\section{DISCUSIÓN Y CONCLUSIONES}

La revisión sistemática efectuada en esta investigación ha permitido identificar algunos estudios causales, durante la última década y sin sesgo de publicación, es decir todos los artículos fueron publicados, dada la importancia de sus resultados, donde la noción de capital social ha atraído un enorme interés de investigación en la interfaz de la economía y las ciencias sociales. Encontrándose, que el capital social ha sido definido por varios autores como el conjunto de normas de confianza interpersonal, valores, actitudes, redes, satisfacción en la vida y apoyo al cambio entre personas e instituciones en una sociedad, que define el grado de asociatividad entre los diferentes actores sociales y facilita acciones colectivas y de cooperación.

Entonces, el capital social implica redes o puentes sociales con otras personas, por lo que viajar en vehículos de transporte público o esperar en paradas o intercambios, fomenta la interacción social con personas que no se conocen y esto probablemente sea un objetivo principal de los servicios de transporte que buscan mejorar el capital social. En este sentido, Canitez (2019) indica que el capital social puede aliviar los problemas de acción colectiva y llenar el espacio vacío dejado por las instituciones formales del transporte público en los países en desarrollo. 
Sin embargo, la valoración directa entre el capital social, el transporte público, y la movilidad sostenible fue compleja, sustancial y débil, coincidiendo con Currie \& Stanley (2008). Aunque, el transporte público ofrece beneficios económicos y sociales obvios, como un medio para aumentar el acceso a las redes sociales de los desfavorecidos, se ha demostrado que fomentan el desarrollo sostenible, al igual que el bienestar social, la productividad y las redes sociales. Igualmente, hace falta mejorar las opciones de movilidad sustentable a nivel mundial y en Venezuela no es la excepción, lo que debilita el capital social, sobre todo en comunidades desfavorecidas.

El transporte público, por definición, significa viajar con otros, y fomentar la interacción social o copresencia durante el viaje, pues esté crea la oportunidad de formar el llamado capital social puente. Al planificar el transporte público, es importante no excluir a ciertos grupos, como las personas con un nivel socioeconómico bajo o personas con discapacidad. Una mala planificación del transporte, puede conducir a aislamiento social para estos grupos. No obstante, la dependencia del automóvil crea un deterioro en el capital social.

El concepto de capital social es nuevo en la movilidad social y en el campo de la accesibilidad. Las personas viajan para lograr importantes logros personales, económicos y sociales. Por otro lado, se requiere de una movilidad virtual en el transporte público para promover la inclusión financiera y aumentar el capital social con herramientas como: aplicaciones de pago, billeteras electrónicas, pagos con QR, así como la anunciada posibilidad de usar las tarjetas de crédito o débito por puntos inalámbricos u otros.

El Sistema de Transporte Público en Venezuela es insuficiente y negativo, lo que disminuye el capital social y la movilidad sustentable, ya que no cuenta con una planificación urbana acorde a los nuevos tiempos y permanente, falta de integración holística entre los diferentes medios de transporte existentes en el país, con los diferentes actores, operadores, autoridad pública y usuarios, a fin de lograr un sistema moderno, seguros, asequibles, accesibles y sostenibles para todos, convirtiéndose en ciudades habitables, en particular mediante la ampliación del transporte público, prestando especial atención a las necesidades de las personas en situación de vulnerabilidad o grupos desfavorecidos, para que se trasladen desde un origen hasta su destino, de forma competitiva, con un servicio de buena calidad y no empírica.

Por último, las fuentes consultadas evidencian que el capital social de Venezuela es negativo y precario, por ende, representa un serio obstáculo para la orientación del país hacia la movilidad sustentable. La sociedad está atomizada y fracturada en muchos aspectos, aunado a los datos analizados del Informe LATINOBARÓMETRO (2018), como son la mala situación económica actual del país, bajos índices de confianza interpersonal, la satisfacción con la vida ha disminuido, y en cuanto a la clase social, predomina la clase baja, entre otros.

Por otro lado, hay una falta de cantidad de investigación primaria cuantitativa asociada con la medición del capital social, abriendo posibilidades insospechadas de investigación en materia de: planificación del transporte urbano, políticas públicas, innovación del transporte público, movilidad social y virtual, entre otros.

\section{Declaración responsable y conflicto de intereses}

La autora declara no tener ningún conflicto de interés potencial con respecto a la investigación, autoría y / o publicación de este artículo.

\section{REFERENCIAS}

Abraham, A. (1985). Subsistence Credit: Survival Strategies among Traditional Fishermen. Economic and Political Weekly, 20(6), 247-52

Axhausen, K. (2005). Redes sociales y viajes: Algunas hipótesis. K. Donaghy, S. Poppel reuter.

Banco Mundial (2001). Juntos podemos niveles y determinantes del capital social de Argentina. http://documents1.worldbank.org/curated/es/982731468201587870/pdf/242810SPANISH01ates0del0CS01PUBLIC1.pdf

Bowles, S. (1999). Social Capital and Community Governance. Focus, 20(3), 6-10. 
Bunting, M. (2004). Making Public Transport Work. McGill-Queen's University Press.

Burt, R. (1992). The Contingent Value of Social Capital. Administrative Science Quarterly 42, 339-365.

Canitez, F. (2019). Urban public transport systems from new institutional economics perspective: a literature review. Transport Reviews, 39(4), 511-530. https://doi.org/10.1080/01441647.2018.1552631

Carciente, S. \& Varnagy, D. (2005). Capital financiero y capital social: Dos ingredientes del Desarrollo. Líder: revista labor interdisciplinaria de desarrollo regional,13, 13-30

Cass, N., Shove, E., \& Urry, J. (2005). Social Exclusion, Mobility and Access. The Sociological Review, 53(3), 539-555. https:// doi.org/10.1111/j.1467-954X.2005.00565.x

Collier,P \& Willen, J. (1999). Explainig African Economic Performance. Journal of Economic Literature, XXXVII, 64-111.

Currie, G., \& Stanley, J. (2008). Investigating Links between Social Capital and Public Transport. Transport Reviews, 28(4), 529-547. https://doi.org/10.1080/01441640701817197

De Rus, G., Campos, J. \& Nombela, G. (2003). Economía del transporte. Antoni Bosch Editor. Universidad de las Palmas de Gran Canarias.

Easterly, W. (2000). Happy Societies. The Middle Class Consensus and Economic Development. Development Research Group. World Bank

Fukuyama, F. (1995). Trust: The Social Virtues and the Creation of Prosperity. Free Press.

Grootaert, C. \& Narayan, D. (2000). Local Institutions, Poverty and Household Welfare in Bolivia. Local Level Institutions. Documento de trabajo 9. World Bank, Social Development Department.

República Bolivariana de Venezuela (2008). Ley de Transporte Terrestre. Gaceta Oficial Nº-38.985.

Mattisson, K., Håkansson, C., \& Jakobsson, K. (2015). Relationships Between Commuting and Social Capital Among Men and Women in Southern Sweden. Environment \& Behavior, 47(7), 734-753. https://doi.org/10.1177/0013916514529969

Ministerio del Poder Popular para Transporte y Obras Públicas (2018, 01 de noviembre). Ministro Ing. Hipólito Abreu presidió la instalación del Órgano Superior de la Misión Transporte en el estado Vargas. http://www.mppt.gob.ve/2018/ministro-ing-hipolito-abreu-presidio-la-instalacion-del-organo-superior-de-la-mision-transporte-en-el-estado-vargas/

Ministerio del Poder Popular para Transporte y Obras Públicas (2016). Misión Transporte. http://www.mpptop.gob.ve/ mision-transporte/

Molinero, M. \& Sanchez, A. (1998). Transporte Publico: Planeación, Diseño, Operación y Administración. Fundación ICA.

Moser, C. (1996). Confronting Crisis: A Comparative Study of Household Responses to Poverty and Vulnerability in Four Poor Urban Communities. Environmentally and Socially Sustainable Development Studies and Monographs Series 8. World Bank.

Narayan, D. \& Lant, P. (1999). Cents and sociability: household income and social capital in rural Tanzania. Economic Development and Cultural Change, 47(4), 871-897.

Östh, J., Dolciotti, M., Reggiani, A., \& Nijkamp, P. (2018). Social Capital, Resilience and Accessibility in Urban Systems: a Study on Sweden. Networks \& Spatial Economics, 18(2), 313-336. https://doi.org/10.1007/s11067-017-9375-9

Padrón, C. (2020). Impacto Económico y Social de los Vehículos de Carga "Perreras" en Venezuela con énfasis en Vargas. Revista Caribeña de Ciencias Sociales. https://www.eumed.net/rev/caribe/2020/06/transporte-urbano-venezuela.html

Petticrew, M. \& Roberts, H. (2006). Systematic Reviews in the Social Sciences: a practical guide BLACKWELL PUBLISHING. Hard cover: Alk. paper.

Portes, A. (1998). Social capital: its origins and applications in modern Sociology. Annual Review of Sociology, 24(1), pp. 1-24.

Preston, H. \& Raje, F. (2006). Accessibility, mobility and transport-related social exclusion. Journal of Transport Geography. https://doi.org/ 10.1016/j.jtrangeo.2006.05.0

Putnam, R. (1993). Making Democracy Work: Civic Traditions in Modern Italy. Princeton University Press, Princeton.

Putnam, R. (2000). The prosperous community. Social capital and public life. The American Prospect, 4 (13), $35-42$.

Programa Venezolano de Educación Acción en Derechos Humanos (2016). Transporte público, accesible y de calidad, para todas y todos. Una propuesta ante el aumento de la gasolina. https://www.derechos.org.ve/web/wp-content/uploads/ transportepublico-copia.pdf

República Bolivariana de Venezuela (1998, 26 de junio). Reglamento de la Ley de Tránsito Terrestre República Bolivariana de Venezuela. Gaceta Oficial No. 5.240, 26 de junio de 1998. Caracas: Venezuela.

Rezeanu, C.I., Briciu, A., Briciu, V., Repanovici, A., \& Coman, C. (2016). The Influence of Urbanism and Information Consumption on Political Dimensions of Social Capital: Exploratory Study of the Localities Adjacent to the Core City from Brașov Metropolitan Área, Romania. PLoS ONE, 11(1), 1-24. https://doi.org/10.1371/journal.pone.0144485 
Seligman, A. (1997). The Problem of Trust. Princeton University Press.

Shove, E. (2002). Rushing Around: Coordination, Mobility And Inequality. Draft paper for the Mobile Network Meeting, Department Sociology. Lancaster University. https://www.lancaster.ac.uk/staff/shove/choreography/rushingaround.pdf

Stanley, J. \& Currie, G. (2006). Social Capital, Community Strengthening and Public Transport Stage 1-Methodology Development Project. Institute of Transport Studies, Monash University.

Temple, J. (1998). initial conditions, social capital and growth in Africa. Journal of African Economies, 7(3), $309-347$.

Urry, J. (2002). Mobility and proximity. Sociology, 36(2), 255-274.

Von Mentz, B. (2003). Movilidad Social de Sectores Medios en México. Una Retrospectiva Histórica (Siglos XVIII al XX). Centro de Investigación y Estudios Superiores en Antropología Social.

Vuchic, V. (2000). Transportation for Livable Cities. Centre for Urban Policy Research Press. 